\title{
Automated Recognition of Lung Diseases in CT images based on the Optimum-Path Forest Classifier
}

\author{
Pedro P. Rebouças Filho • Antônio C. da Silva Barros • Geraldo L. B. \\ Ramalho - Clayton R. Pereira · João Paulo Papa - Victor Hugo C. de \\ Albuquerque · João Manuel R. S. Tavares
}

Received: date / Accepted: date

\begin{abstract}
The World Health Organization estimated that around 300 million people have asthma, and 210 million people are affected by Chronic Obstructive Pulmonary Disease (COPD). Also, it is estimated that the number of deaths from COPD increased $30 \%$ in 2015 and COPD will become the third major cause of death worldwide by 2030. These statistics about lung diseases get worse when one considers fibrosis, calcifications and other diseases. For the public health system, the early and accurate diagnosis of any pulmonary disease is mandatory for effective treatments and prevention of further deaths. In this sense, this work consists in using information from lung images to identify and classify lung diseases. Two steps are required to achieve these goals: automatically extraction of representative image features of the lungs, and recognition of the possible disease using a computational classifier.
\end{abstract}

Pedro P. Rebouças Filho, Antônio C. da Silva Barros, Geraldo L. B. Ramalho

Laboratório de Processamento Digital de Imagens e Simulação Computacional, Instituto Federal de Federal de Educação, Ciência e Tecnologia do Ceará (IFCE), Ceará, Brazil. E-mail: pedrosarf@ifce.edu.br, carlosbarros@lapisco.ifce.edu.br, gramalho@ifce.edu.br

Clayton R. Pereira, João Paulo Papa

Departamento de Ciência da Computação, Universidade Estadual Paulista, Bauru, São Paulo, Brazil. E-mail: claytontey@gmail.com,papa@fc.unesp.br

Victor Hugo C. de Albuquerque

Programa de Pós-Graduação em Informática Aplicada, Universidade de Fortaleza, Fortaleza-CE, Brazil. E-mail: victor.albuquerque@unifor.br

João Manuel R. S. Tavares

Instituto de Ciência e Inovação em Engenharia Mecânica e Engenharia Industrial, Departamento de Engenharia Mecânica, Faculdade de Engenharia, Universidade do Porto, Porto, Portugal. E-mail: tavares@fe.up.pt (corresponding Author)
As to the first step, this work proposes an approach that combines Spatial Interdependence Matrix (SIM) and Visual Information Fidelity (VIF). Concerning the second step, we propose to employ a Gaussian based distance to be used together with the Optimum-Path Forest (OPF) classifier to classify the lungs under study as normal or with fibrosis, or even affected by COPD. Moreover, to confirm the robustness of OPF in this classification problem, we also considered Support Vector Machines and a Multilayer Perceptron Neural Network for comparison purposes. Overall, the results confirmed the good performance of the OPF configured with the Gaussian distance when applied to SIM and VIF based features. The performance scores achieved by the OPF classifier were as follows: average accuracy of $98.2 \%$, total processing time of 117 microseconds in a common personal laptop, and $F$-score of $95.2 \%$ for the three classification classes. These results showed that OPF is a very competitive classifier, and suitable to be used for lung disease classification.

Keywords Medical Imaging - Optimum-Path Forest . Feature Extraction · Image Classification.

\section{Introduction}

Since its establishment in 1948, the World Health Organization (WHO) is responsible for ranking the most dangerous diseases, which are led today by ischaemic heart disease followed by cerebral vascular accidents, usually known as strokes. Additionally, the large number of lung diseases that affect the worldwide population has also been confirmed by WHO [1]. Therefore, research in the field of Pulmonology has become of great importance in public health, and it has been mainly fo- 
cused on asthma, bronchiectasis and Chronic Obstructive Pulmonary Disease (COPD) [2, 3].

WHO estimated around 300 million people suffering from asthma, being this disease in charge of around 250 thousand deaths per year worldwide [4. In addition, WHO estimated that around 210 million people are affected by COPD, which is responsible for about 3 million deaths in 2015 (5\% of all worldwide deaths in that year) [5]. Recent studies revealed that COPD is mainly present in the 20-to-45-year-old age bracket, although it is usually characterized as an over-50-year-old disease. Accordingly, WHO estimated that the number of deaths due to COPD increased 30\% in 2015, being such disease the third cause of mortality worldwide by 2030 6].

Based on the aforementioned context, the early and accurate diagnosis of pulmonary diseases is mandatory for the public health systems in order to attain effective treatments and prevention of further deaths. From a clinical viewpoint, aid diagnosis tools and systems are of great importance for specialists to improve people health. In this sense, this work uses information from lung images to identify and classify lung diseases according to two main steps: (i) extraction of lung image features, and (ii) identification of lung diseases using a machine learning classifier. As to the extraction of information from the lungs under study, the Computed Tomography (CT) imaging modality has been commonly used in pulmonology for diagnostic purpose, since it enables the analysis of the lungs and their internal structures with high quality and accuracy [7, 8, 9, 10. However, for the effective pulmonary disease analysis and diagnosis from CT images, it is usually necessary to segment the regions of interest (ROIs), i.e., the lungs in the input images, since these ROIs must include the lung structures that are the object of study [11, 12, 8].

The segmentation step proposed in this work is based on the Adaptive Crisp Active Contour Models (ACACM) method, which uses an active contour model specially designed for medical imaging that has outperformed traditional image segmentation methods, such as Watershed, Region Growing, Mathematical Morphology, as well as the well-known Active Contour algorithm [8, 13. Following the segmentation step, an automatic procedure can be applied to detect diseases in lung CT images in order to support radiologic diagnosis. Some studies have yielded promising disease detection results, as the one reported in [14, where texture descriptors extracted from a Gray Level Co-occurrence Matrix (GLCM) [15] are used to distinguish three disease patterns (nodule, emphysema and frosted glass) relatively to the normal one. GLCM texture descriptors are also employed in [16] to determine whether lungs are healthy or not. Furthermore, some works have been proposed to address the detection of certain specific lung diseases in images, such as nodules [17] and emphysema [18, 8, just to name a few. Finally, Ramalho et al. [16] proposed a feature extraction method based on a Spatial Interdependence Matrix (SIM) to classify lung diseases. In this work, this approach is extended by combining Visual Information Fidelity (VIF) [19] with attributes obtained from the SIM method.

In the classification step, we focused our study on a recent classifier known as Optimum Path Forest (OPF) 20, 21, 22, which has gained increasing attention in the last years because it presents interesting characteristics: (i) it is free of hard-tocalibrate control parameters; (ii) it does not assume any shape/separability of the feature space; (iii) it runs the training phase usually much faster than other techniques; and (iv) it can make decisions based on global criteria. Moreover, the OPF classifier does not interpret the classification task as a hyperplane optimization problem, but as the computation of optimum paths from key patterns (known as prototypes) to the remaining nodes. As such, each prototype becomes a root from its optimum-path tree, and then each node is classified according to its strongest connected prototype. This process defines a discrete optimal partition (influence region) of the feature space. Therefore, due its efficiency and effectiveness, combined with its parameter independence and robustness to highly non-linear datasets, the OPF classifier can be very effective in the automated classification of CT based images of lungs as being healthy or affected by diseases like COPD or fibrosis.

OPF has been used in different application domains, such as Biomedical engineering, for example, for the classification of electroencephalography (EEG) 23] and electrocardiography (ECG) 24] signals, classification of human intestinal parasites [25], and classification of brain tissue in Magnetic Resonance images [26], with very promising performances [27, 28, 29, 30, 31, 32, 33, mainly with respect to its computational efficiency. As to the classification rates, OPF has also obtained notable results, being in many cases at least as good as the ones obtained by some traditional classifiers, such as Support Vector Machines (SVMs), Bayesian classifiers, Artificial Neural Networks (ANNs) and $k$-nearest neighbors $(k-\mathrm{NNs})$ 34, 35, 36, 37, 38, 39, 29, 40, 41, 42, 43, 27, 28, 31, 30, 32, 25, 26, 33, 23, 44.

In previous works [45, 46, 47, 48, 9, 49, 13, 50, the authors have evaluated the potential of different segmentation approaches in order to tackle the automatic detection of lung nodules in images [51. Overall, the results achieved shown that the studied techniques were 
competitive in terms of accuracy and processing time. However, the choice of the feature extractor is a really critical decision in order to efficient results can be achieved. Additionally, our previous works let us conclude that the automated classification using machine learning techniques, especially using the Optimum Path Forest classifier [40, 34, 35, 37, 38, 39, 41, 42, 43, is a very promising tool in medical assisted diagnosis.

However, despite the number of current applications based on the OPF classifier, we have not observed any work related to pulmonary disease identification so far. This article also addresses the detection of fibrosis and emphysema diseases, which are the COPD main components, based on a new feature extraction method (SIM). A classification experiment was also performed to compare the performance achieved from the SIM and GLCM texture based descriptors. The VIF index 19] was also investigated, since it performs similarly to SIM based based descriptors. In fact, both approaches are based on the analysis of a reference image. As a final contribution of this article, we considered to employ a Gaussian based distance to weight OPF edges, since the original approach uses the Euclidean distance [52. For evaluation purpose, the computational cost (processing time), accuracy rate (success rate), positive predictive value (precision), sensitivity (recall) and $F$-measure were used to calculate the similarity among the patterns under classification [44, as well as to assess the features extracted from the lung-segmented CT images.

The remainder of this article is organized as follows. Sections 2 and 3 present the methodology and a theoretical background about the OPF classifier, respectively. Section 4 discusses the experimental results, and Section 5 states the main conclusions and possible future works.

\section{Methodology}

\subsection{Dataset}

The images included in the used experimental dataset were acquired using the following CT systems: Toshiba Aquilion (TA), GE Medical system LightSpeed16 (GEMS and Phillips Brilliance 10 (PB). All images have a resolution of $512 \times 512$ pixels, a bit depth of 16 bits, and were obtained in partnership with the Walter Cantídio Hospital of the Federal University of Ceará in Brazil [2, 3. This research was approved and evaluated by the Research Ethics Committee - COMEPE (Protocol number $35 / 06$ ), and complied with the requirements of Resolution number 196/96 of the National Health Council concerning the research in human beings [2, 3].
The CT lung images were acquired on apex, hilum and on the base of the axial plane, according to the following conditions: each slice has $1.5 \mathrm{~mm}$ of thickness, the field of view was $312 \mathrm{~mm}$, the electrical tension in the tube was $120 \mathrm{kV}$, and the electric current was 200 $\mathrm{mA}$; lung window adjustment: the level and width were -600 and $1600 \mathrm{HU}$, respectively; the dimension of the reconstructed window was $512 \times 512$ pixels, and the voxels have dimensions of $0.585 \times 0.585 \times 1.5 \mathrm{~mm}$, being quantified in 16 bits. In this study, we used $36 \mathrm{CT}$ images: 12 from healthy volunteers, 12 from patients with fibrosis, and 12 from patients with COPD, resulting in 72 lungs, since each image contained two lungs. Concerning these lungs, 27 were healthy, 21 affected by fibrosis and 24 had COPD.

Rebouças Filho et al. 8] employed the ACACM segmentation method to detect the inner region of the lungs in CT images, being the segmented regions used to obtain the attributes employed in the classification of the lungs under study. The ACACM method was adopted here because it provides accurate segmentation results; indeed, it outperformed other segmentation methods available in the literature based on region growing, watershed, mathematical morphology and traditional active contour techniques [53,54, both in terms of processing time and accuracy. This method is based on an Active Contour Model, and encompasses computational intelligence techniques with prior knowledge about the lung anatomy [55, 8, 56, 57. Figure 1 depicts examples of lung segmentation results in thorax CT images acquired from patients with COPD and fibrosis, as well as from a healthy volunteer.

\subsection{Feature extraction}

The first feature extraction method employed in this work was the GLCM matrix suggested in [15] and often adopted in problems related to texture analysis [58, 59]. Figure 2 illustrates a three-dimensional distribution of the samples achieved by the GLCM built upon the experimental image dataset used in this work. This representation was built considering the best set of features ) by means of the Principal Component Analysis (PCA) technique.

The second feature extraction method, namely the Spatial Interdependence Matrix, uses co-occurrence statistics to analyze the structural information based on the way the Human Visual System interprets scenes. Therefore, this method is commonly used to assess image structural degradation. Moreover, it performs quite similarly to the VIF index [19, which assesses differences between an image and its degraded version. 


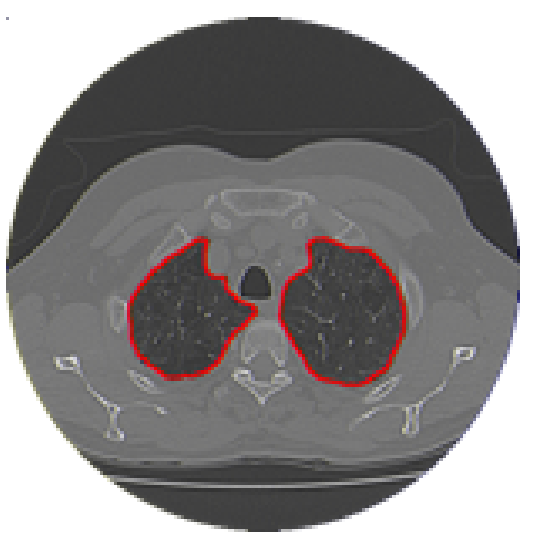

(a)

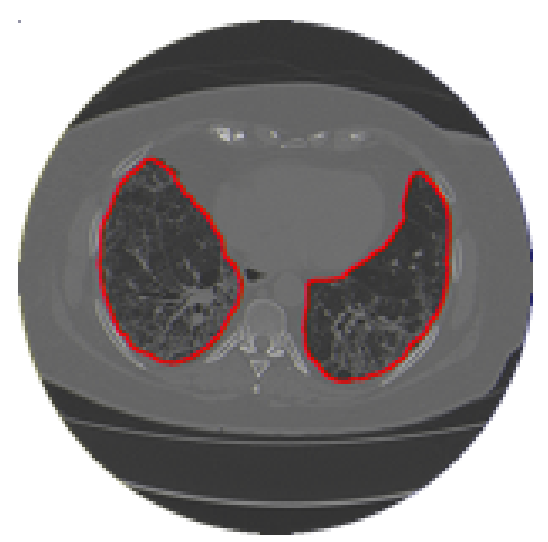

(b)

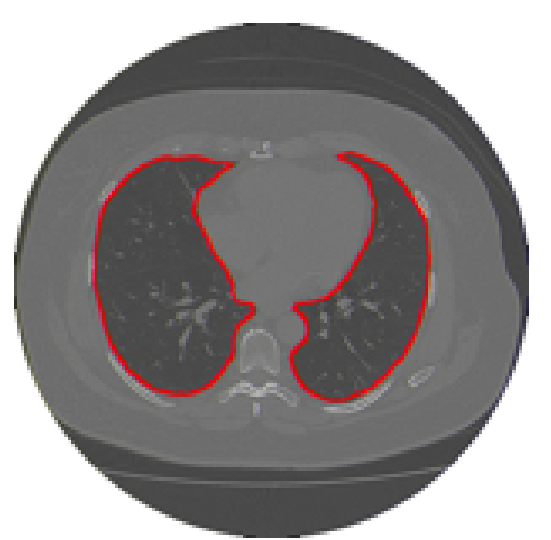

(c)

Fig. 1: Examples of segmentation results for thorax CT images with: a) COPD, b) fibrosis and c) healthy lungs [16].

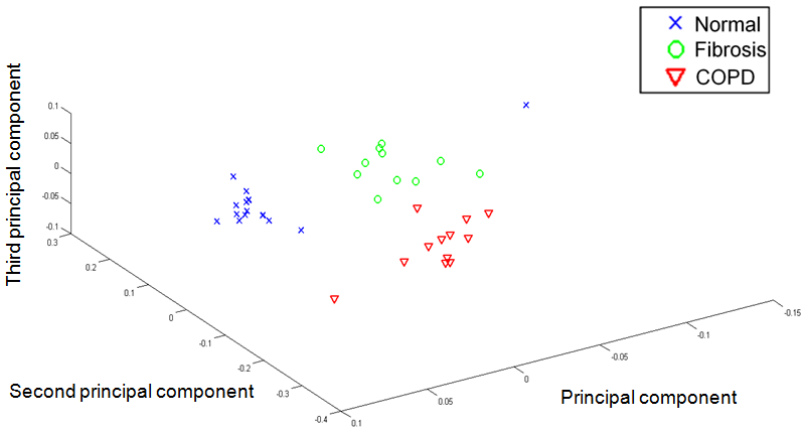

Fig. 2: Three-dimensional distribution concerning three classes by applying PCA on the GLCM built from the image dataset under study.

Let us consider an image $I$ and its degraded image $J$ as a set of gray levels in domain $D \subset Z^{2} \in$ $\{0,1,2, \ldots, N\}$, where $N$ is the total number of gray levels. One can arrange the transitions among the intensities of the pixels that are spatially related in both images into an $(N \times N)$ matrix whose elements $M_{i j}$ are defined as follows:

$M_{i j}=|\{(i, j): i=j, i=I(p), j=J(p)\}| \quad \forall_{p} \in D$,

which is basically the number of times the intensities $i=I(p)$ and $j=J(p)$ of pixels $p$ from images $I$ and $J$ verify some spatial constraint.

Three structural attributes obtained using the SIM technique are commonly used to assess the degraded image $J$ relatively to the original one $I$ : correlation (Cor), inverse difference moment $(I d m)$ and chi-square $(C h i)$ [16. These attributes represent the level of degradation from three different perspectives: structural similarity, structural degradation and structural independence, respectively.
In order to calculate the structural attributes Cor, $I d m$ and $C h i$, a symmetric version of matrix $M: M_{S}=$ $\left(M+M^{T}\right) / 2$ is used. Then, a normalization procedure is applied to $M_{S}$, such that $\sum M_{S}=1$, in order to obtain the weight of each transition-pair as an approximation of probabilities. Afterwards, the three structural attributes are computed as follows:

Cor $=\sum_{i, j=0}^{N-1} \frac{\left(i-\mu_{i}\right)\left(j-\mu_{j}\right) M_{i j}}{\sqrt{\rho_{i}^{2} \rho_{j}^{2}}} \epsilon[-1,1]$,

$I d m=\sum_{i, j=0}^{N-1} \frac{M_{i j}}{1+|i-j|} \epsilon[0,1]$,

$C h i=\sum_{i, j=0}^{N-1} \frac{\left(O_{i}-E_{i}\right)^{2}}{E_{i}} \epsilon[0,1]$,

where $\mu_{i}$ and $\mu_{j}$ stand for the average values of line $i$ and column $j$ of matrix $M_{S}$, and $\rho_{i}$ and $\rho_{j}$ are the standard deviation of each line $i$ and column $j$ of the same matrix; $O_{i}$ refers to the observed weights in the main diagonal $(i=j)$ of $M_{S}$, and $E_{i}$ refers to the expected weights in the main diagonal of $M_{S}$.

The SIM technique provides a visual pattern useful to interpret the degraded image. When the image structures are not degraded, the weights are well distributed closely to the diagonal of the matrix. Otherwise, different patterns appear according to the structural degradation. Therefore, the SIM pattern of a healthy lung is quite different from a fibrosis one. The structures of a healthy lung are small, sparse and have high contrast. However, the fibrosis structures are spread through the lung region. 
The degradation model used in this work relies on the knowledge that $\mathrm{CT}$ imaging systems usually blur the lung structures. Thus, the input images were smoothed by convolving them with a $(3 \times 3)$ Gaussian kernel with zero mean and unitary standard deviation. Our experiments have shown that this convolution mask is sufficient to smooth small structures, and has low impact on the overall computational cost. The number of gray-levels $N$ was set to 64 in the computation of the SIM descriptor, which ensures the successful detection of structural changes and good numerical estimations for the co-occurrence frequencies.

\subsection{Lung disease classification}

Usually, COPD images present low-medium intensity and large structures. On the other hand, pulmonaryfibrosis (PF) lung images present particular texture, and therefore are more susceptible to structural changes caused by blurring effects. In fact, the $I d m$ and $C h i$ attributes can easily detect this structural degradation. On the other hand, the fibrosis structures are largely distributed in the lungs and present relative larger dimensions in comparison to protruding structures like blood vessels. More importantly, the Cor attribute value exceeds the $I d m$ and $C h i$ ones for degraded structures in fibrosis images. Regarding healthy lung (HL) images, usually they are more uniform than PF and COPD images. Nevertheless, HL images present some prominent vessels degraded by the smoothing filter. In general, the SIM attributes for HL are lower than the ones calculated for PF and COPD images.

The proposed lung disease descriptor is a set of three attributes in a vector $A=\{C o r, I d m, 1-C h i\}$ extracted from the SIM features computed from the 72 lung images under study. Further, the dataset of descriptors consists of 27 vector samples of healthy lungs, 24 of COPD and 21 of pulmonary fibrosis, respectively. Experts on pulmonary diseases provided the gold standard (GS) reference labels that were used to training and validate the artificial classifiers. Figure 3 illustrates the projection of both sets of descriptors in the bi-dimensional space using an U-Matrix projection [60. This n-dimensional visualization tool reveals the discriminant power of the descriptors under analysis through a distance map that indicates how close is an entity to its neighbors that belong to the same class. The color intensity in Figures $3 \mathrm{a}$, 3b and 3c is proportional to the distance, i.e., the darker the color, the closer the entity is to the neighbors in the same class.

Figures 3d, 3e and 3flillustrate these U-Matrices using color labelling for the data samples. Two classes are well discriminated when there is a well-delimited light region among them. Therefore, this map provides a visual interpretation of the spatial arrangement of the samples in clusters of similar meaning. One can observe that the SIM U-Matrix presents the best discrimination due to the presence of three well-defined regions, one for each class. The GLCM U-Matrix presents more than three regions, which means there are samples associated, i.e., belonging to different classes. On the other hand, the VIF U-Matrix presents only two well-defined regions, being not so useful to discriminate the three classes involved. From the images, one can observe that both GLCM and VIF descriptors performed poorly in the present context. However, it is noteworthy the texture descriptors were able to provide a good discrimination of the COPD cases [16].

Figure 4 displays a diagram that highlights the boundaries found among classes in the segmented lung images and their associated SIM. The matrices exhibit a particular pattern for each lung image, as well as similarities among samples that belong to the same class. The largest dispersion around the diagonal indicates that high contrasted structures are degraded, as happened in the COPD images. The matrices show a similar pattern region with a "V" shape in the HL images, indicating the imaged lungs present high contrast among adjacent structures [16]. 


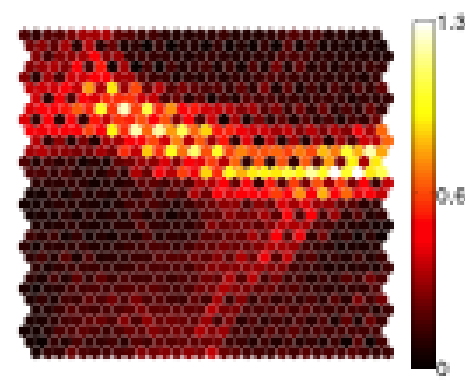

(a)

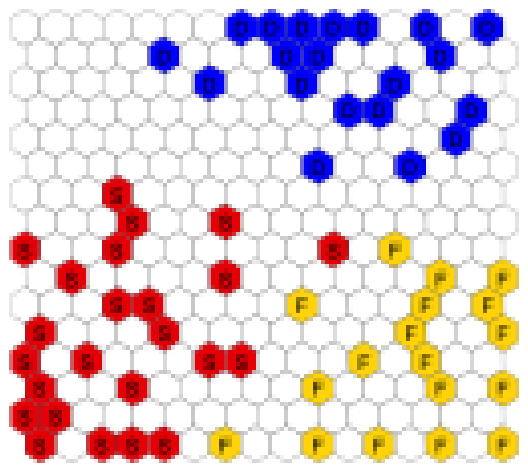

(d)

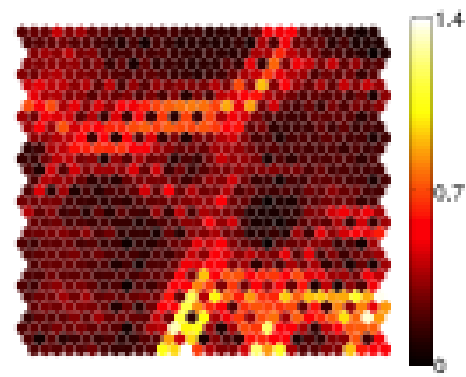

(b)

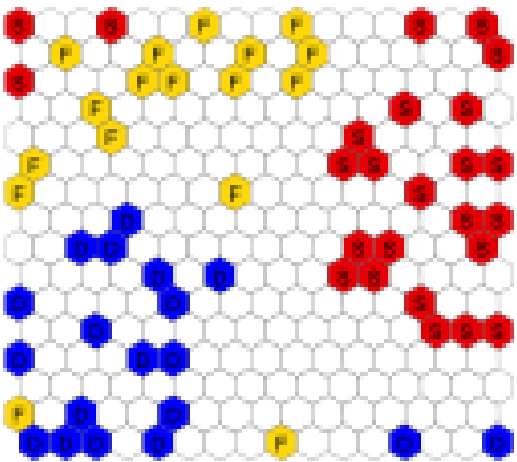

(e)

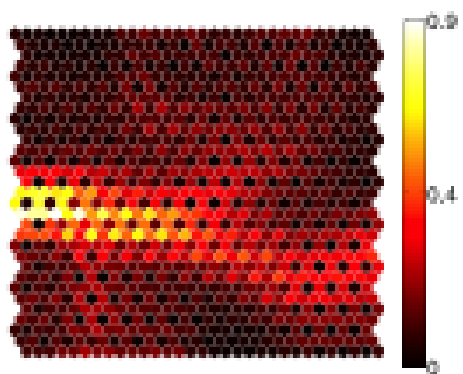

(c)

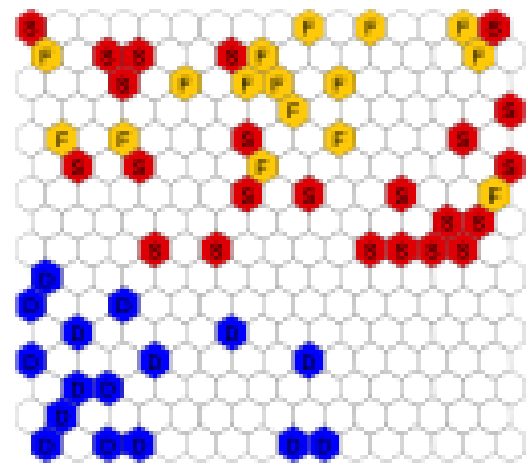

(f)

Fig. 3: (a-c) U-Matrices for the SIM, GLCM and VIF descriptors used in lung disease discrimination; (d-f) the colors identify the HL (red), PF (yellow), and COPD (blue) classes 16.

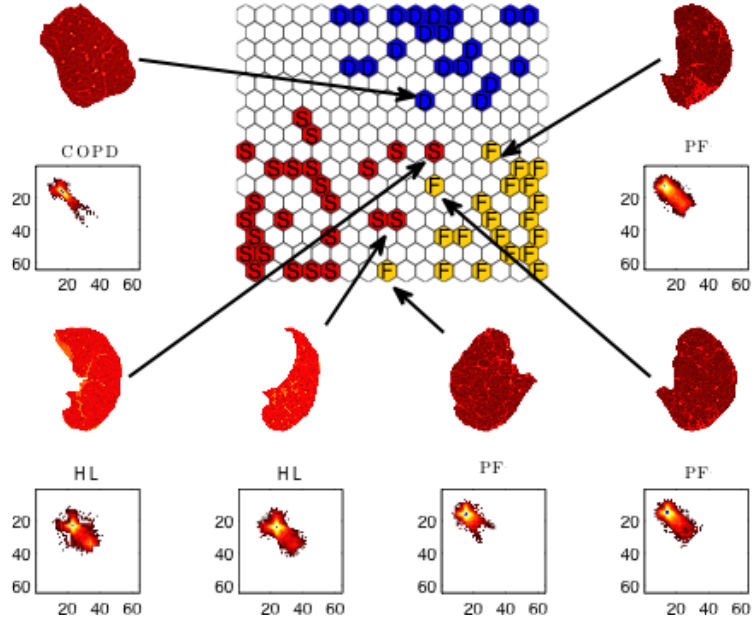

Fig. 4: Lung images associated to the boundaries of the classes under study. The colors in the U-Matrix identify the HL (red), PF (yellow) and COPD (blue) classes [16].

\section{Optimum-path Forest classifier}

The OPF classifier works by modeling the problem of pattern recognition as a graph partition in a given feature space. The nodes are represented by feature vec- tors and the edges connect all pairs of them, defining a full connectedness graph. This kind of representation is straightforward, given that the graph does not need to be explicitly represented, allowing the saving of memory. The partition of the graph is carried out by a competition process between key samples (prototypes), which defines optimum paths to the remaining nodes of the graph. Each prototype sample defines its optimumpath tree (OPT), and the collection of all OPTs defines an optimum-path forest, which gives the name to the classifier [61].

Let $Z=Z_{1} \cup Z_{2}$ be a dataset labeled with a function $\lambda$, in which $Z_{1}$ and $Z_{2}$ are, respectively, a training set and a test set such that $Z_{1}$ is used to train the classifier and $Z_{2}$ is used to assess its accuracy. Also, let's $S \subseteq Z_{1}$ be a set of prototype samples. Essentially, the $\mathrm{OPF}$ classifier creates a discrete optimal partition of the feature space such that any sample $s \in Z_{2}$ can be classified according to this partition. The found partition is the OPF computed in $\Re^{n}$ by the image foresting transform (IFT) algorithm 62].

The OPF algorithm may be used based with any smooth path-cost function that can group samples with similar properties 62. In this work, we considered the 
path-cost function $f_{\max }$, which is computed as follows:

$$
\begin{aligned}
f_{\max }(\langle s\rangle) & = \begin{cases}0 & \text { if } s \in S, \\
+\infty & \text { otherwise },\end{cases} \\
f_{\max }(\pi \cdot\langle s, t\rangle) & =\max \left\{f_{\max }(\pi), d(s, t)\right\},
\end{aligned}
$$

in which $d(s, t)$ means the distance between samples $s$ and $t$, and a path $\pi$ is defined as a sequence of adjacent samples. Notice that $\langle s\rangle$ stands for a trivial path rooted at sample $s$, and $\langle s, t\rangle$ denotes the arc between the adjacent nodes $s$ and $t$.

Therefore, one has that $f_{\max }(\pi)$ computes the maximum distance between adjacent samples in $\pi$, when $\pi$ is not a trivial path. The OPF algorithm assigns one optimum path $P^{*}(s)$ from $S$ to every sample $s \in Z_{1}$, establishing an optimum path forest $P$ (a function with no cycles that assigns to each $s \in Z_{1} \backslash S$ its predecessor $P(s)$ in $P^{*}(s)$ or a marker nil when $\left.s \in S\right)$. Let's $R(s) \in S$ be the root of $P^{*}(s)$ that can be reached from $P(s)$. Then, OPF computes for each $s \in Z_{1}$, the $\operatorname{cost} C(s)$ of $P^{*}(s)$, the label $L(s)=\lambda(R(s))$, and the predecessor $P(s)$.

The OPF classifier is composed of two distinct phases: (i) training and (ii) classification. The former step consists, essentially, in finding the prototypes and computing the optimum-path forest, which is the union of all OPTs rooted at each prototype. After that, a sample is taken from the test sample, connected to all samples of the OPF generated in the training phase and then it is found which node offered the optimum path to it. Notice that this test sample is not permanently added to the training set, i.e., it is used only once. The next sections describe in details this procedure.

One can say that $S^{*}$ is an optimum set of prototypes when the OPF algorithm minimizes the classification errors for every $s \in Z_{1}$. $S^{*}$ can be found based on the theoretical relation between the minimum-spanning tree (MST) and the optimum-path tree for $f_{\max }$ [63]. The training essentially consists in finding $S^{*}$ and an OPF classifier rooted at $S^{*}$.

By computing a MST in the complete graph $\left(Z_{1}, A\right)$, a connected acyclic graph whose nodes are all samples of $Z_{1}$ and the arcs are undirected and weighted by the distances $d$ between adjacent samples is established. The spanning tree is optimum since the sum of its arc weights is minimum in comparison to any other spanning tree in the complete graph. In the MST, every pair of samples is connected by a single path that is optimum according to $f_{\max }$. That is, the minimumspanning tree contains one optimum-path tree for any selected root node. The optimum prototypes are the closest elements of the MST with different labels in $Z_{1}$ (i.e., elements that fall in the frontier of the classes). Al-

gorithm 1 resumes the training procedure for the OPF classifier.

\section{Algorithm 1 - OPF Training Algorithm \\ INPUT: \\ Output: \\ AuXILIARY:

\begin{abstract}
A $\lambda$-labeled training set $Z_{1}$ and a pair $(v, d)$ for feature vector and distance computation. Optimum-path forest $P$, cost map $C$, label map $L$, and ordered set $Z_{1}^{\prime}$.

Priority queue $Q$, set $S$ of prototypes, and
\end{abstract} cost variable $c s t$.}

1. Set $Z_{1}^{\prime} \leftarrow \emptyset$ and compute by $M S T$ the prototype set $S \subset Z_{1}$.

2. For each $s \in Z_{1} \backslash S$, set $C(s) \leftarrow+\infty$.

3. For each $s \in S$, do

4. $\quad C(s) \leftarrow 0, P(s) \leftarrow$ nil, $L(s) \leftarrow \lambda(s)$, insert $s$

5. $[$ into $Q$.

6. While $Q$ is not empty, do

7. $\quad$ Remove from $Q$ the sample s such that $C(s)$ is

8. minimum.

9. $\quad$ Insert $s$ in $Z_{1}^{\prime}$.

10. For each $t \in Z_{1}$ such that $C(t)>C(s)$, do

11. $\quad$ Compute cst $\leftarrow \max \{C(s), d(s, t)\}$.

12. If cst $<C(t)$, then

\begin{tabular}{l|l|l}
13. & If $C(t) \neq+\infty$, then remove $t$ from $Q$. \\
14. & $P(t) \leftarrow s, L(t) \leftarrow L(s), C(t) \leftarrow$ cst.
\end{tabular}

14. $\left[\begin{array}{l}P(t) \leftarrow s, L(t) \\ \text { Insert } t \text { into } Q .\end{array}\right.$

16. Return the classifier $\left[P, C, L, Z_{1}^{\prime}\right]$.

The OPF time complexity for training is $\theta\left(\left|Z_{1}\right|^{2}\right)$, due to the main (Lines 5-13) and inner loops (Lines 8-13) in Algorithm 1, that are executed $\theta\left(\left|Z_{1}\right|\right)$ times each.

\subsection{Classification}

For any sample $t \in Z_{2}$, it is assumed that all arcs are connecting $t$ with samples $s \in Z_{1}$. Considering all possible paths from $S^{*}$ to $t$, is is found the optimum path $P^{*}(t)$ from $S^{*}$ and $t$ is labeled with the class $\lambda(R(t))$ of its most strongly connected prototype $R(t) \in S^{*}$. This path can be incrementally identified by computing the optimum cost $C(t)$ as:

$C(t)=\min \{\max \{C(s), d(s, t)\}\}, \forall s \in Z_{1}$.

Now, let's the node $s^{*} \in Z_{1}$ be the one that satisfies Equation 6 (i.e., the predecessor $P(t)$ in the optimum path $\left.P^{*}(t)\right)$. Given that $L\left(s^{*}\right)=\lambda(R(t))$, the classifier simply establishes $L\left(s^{*}\right)$ as the class of $t$. An error occurs when $L\left(s^{*}\right) \neq \lambda(t)$. Algorithm 2 resumes the OPF classification process.

\section{Algorithm 2 - OPF Classification Algorithm}

INPUT:

Classifier $\left[P, C, L, Z_{1}^{\prime}\right]$, evaluation set $Z_{2}$, and the pair $(v, d)$ for feature vector and distance computation.

Output: Label $L^{\prime}$ and predecessor $P^{\prime}$ maps defined for $Z_{2}$.

Auxiliary: Cost variables tmp and mincost. 


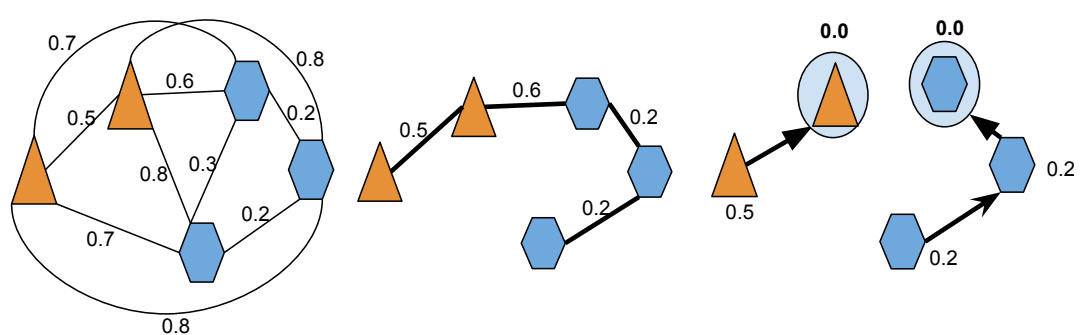

(i)

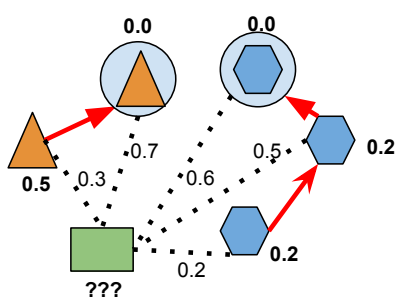

(iv) (ii )

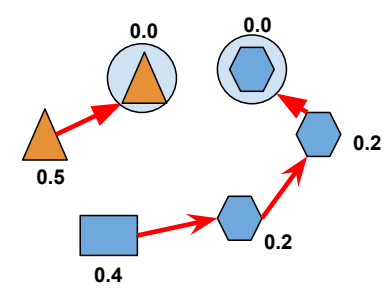

( v)

Fig. 5: (i) Training set modeled as a complete graph; (ii) computation of a minimum spanning tree over the training set; (iii) optimum-path forest found over the training set (prototypes are highlighted); (iv) classification process of a "green" sample; and (v) a test sample is finally classified.

1. For each $t \in Z_{2}$, do

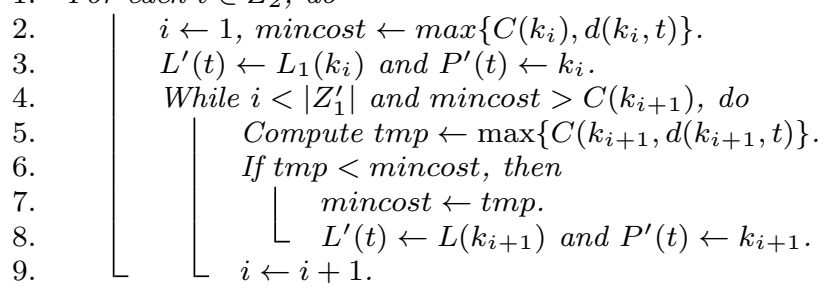

10. Return $\left[L^{\prime}, P^{\prime}\right]$.

In Algorithm 2. the main loop (Lines 1 - 9) performs the classification of all nodes in $Z_{2}$. The inner loop (Lines $4-9$ ) visits each node $k_{i+1} \in Z_{1}^{\prime}, i=$ $1,2, \ldots,\left|Z_{1}^{\prime}\right|-1$ until an optimum path $\pi_{k_{i+1}} \cdot\left\langle k_{i+1}, t\right\rangle$ is established, Fig. 5 .

\subsubsection{OPF with Gaussian distance}

The OPF algorithm estimates prototypes by calculating the path-cost function $f_{\max }$, as given by Equation 5 . The OPF library available freely, which is known as
LibOPF 1 implements seven approaches to calculate the distance $d(s, t)$ between nodes $s$ and $t$ 20, 21].

In this work, we considered a distance between nodes $s$ and $t$ that is based on the Gaussian probability density function $d_{\text {Gaussian }}(s, t)$ [64]:

$d_{\text {Gaussian }}(s, t)=1-\exp \left(-\frac{\|s-t\|}{2 \sigma^{2}}\right)$,

where $\sigma$ is a parameter that controls the smoothness of the Gaussian function, and $\|s-t\|$ stands for the Euclidean distance between nodes $s$ and $t$. Figure 6 depicts the relationship between the Euclidean and the Gaussian distance with $\sigma$ equal to $1,0.5$ and 0.25 concerning two distinct nodes.

In Figure 6, one can observe the smaller is the $\sigma$ value, the more peaked is the Gaussian distance and closer the two nodes are, and that larger $\sigma$ values correspond to smoother decision boundaries. Then, $\sigma$ defines the $f_{\max }$ calculated in Equation 5 not only by the distance between nodes $s$ and $t$, but by taking into

\footnotetext{
1 http://www.ic.unicamp.br/ afalcao/libopf/LibOPF.
} 


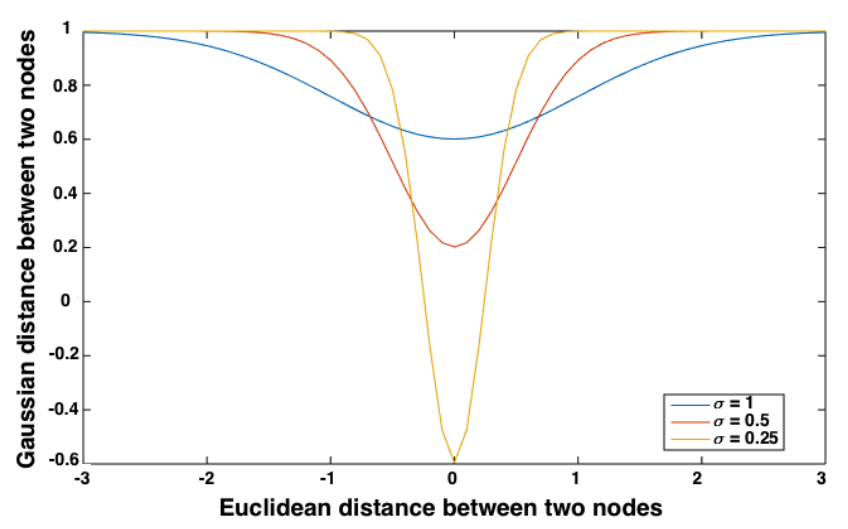

Fig. 6: Examples of the Gaussian distance with $\sigma$ equal to $1,0.5$ and 0.25 .

account the value of the Gaussian distance calculated in Equation 7. As such, close nodes tend to be exponentially more important than the ones farthest away, which may accelerate the learning of OPF and increases its accuracy rate.

\section{Results and Discussion}

In this section, we present the results related to the computational cost and classification accuracy. A personal laptop with an Intel Core i5 at $1.4 \mathrm{GHz}, 4 \mathrm{~GB}$ of RAM and running MAC OS X 10.9.5 was used in all experiments. The classification accuracy was considered as the number of correctly classified samples divided by the total number of samples involved.

The results obtained by the OPF classifier were compared against the ones obtained by a Multi-Layer Perceptron Neural Network (MLP) 65, 66, 67, 36] and Support Vector Machines [68, 69, 70]. Notice all classifiers were implemented in $\mathrm{C}++$. In regard to the SVM based classifier, the Linear, Polynomial, RBF (Radial Basis Function) and Sigmoid kernels were adopted. For the MLP classifier, two topologies were established: one with 3 neurons in the hidden layer, and a second one with 10 neurons in the same layer. Notice that the adopted SVM implementation performs automatically the search for the optimal parameters during the training step.

Firstly, it is necessary to find the $\sigma$ value that fits better to solve Equation 7, as well as to improve the performance of the OPF classifier. Thus, Table 1 indicates the classification accuracy, $F$-score and training and testing times obtained by the proposed approach (i.e., $\mathrm{SIM}+\mathrm{VIF}$ attributes) with the OPF classifier based on the Gaussian distance with $\sigma$ values varying from 0.1 to 0.9 . From Table 1, one can realize that the value of $\sigma$ that led to the best results in terms of accuracy, $F$ score and computational times was 0.9 . Therefore, such value was used in the further experiments and analysis.

Tables 2 and 3 present the average and standard deviation values as to accuracy (Acc), sensitivity (Se) and positive predictive value (PPV) obtained by the OPF, SVM and MLP classifiers considering the features extracted by the GLCM and SIM methods, respectively. The well-known holdout method was used in the experiments, with $50 \%$ of the samples used for training purpose, and the remainder ones employed to test the classifiers over 10 runnings.

From the results presented in Tables 2 and 3 , one can realize the best classification accuracy from the features extracted by the GLCM method was obtained by the OPF classifier using the Euclidean distance (96\%), followed by SVM with linear kernel. Next, the OPF classifier with Chi-Squared measure obtained the second best results $(89.33 \%)$. Using the GLCM based features, the proposed Gaussian distance considering the OPF classifier led to an accuracy of $82.67 \%$. These results are similar to the ones obtained in [16, which are based on Extreme Learning Machine (ELM) techniques applied on SIM based features.

Table 4 indicates the average and standard deviation values as to accuracy (Acc), sensitivity (Se), positive predictive value $(\mathrm{PPV})$ and F-measure $\left(F_{m}\right)$ obtained by OPF, SVM and MLP based classifiers considering SIM+VIF based features. From the results presented in Table 4 , one can confirm that the best classification accuracy achieved from the features extracted using the SIM method plus the ones extracted with the VIF method was obtained by the OPF classifier using the proposed Gaussian distance (i.e., 98.2\%). This accuracy rate was also obtained by the SVM classifier using the RBF kernel. Comparing the data in Tables 2 and 4 . it can be noticed that a gain of accuracy was achieved when the features extracted by the VIF method were also used.

For a complete analysis of the results, non-parametric tests of the variance of the accuracy results of each classifier together with the feature extractors were performed using Friedman's test. This statistical test is used to compare data samples when the same subject is evaluated more than once [71]; it is commonly considered an extension of the Wilcoxon test when it is necessary to use three or more experimental conditions 72. Table 5 shows the statistical test values for the best results obtained using the features extracted using the GLCM and SIM methods when used independently and combined against the ones obtained using the proposed approach, i.e., the SIM+VIF features and OPF with the Gaussian distance. In this table, the training, 
Table 1: Accuracy (Acc), sensitivity (Se), training and testing times obtained from SIM plus VIF attributes using the OPF classifier based on the Gaussian distance with different $\sigma$ values.

\begin{tabular}{lcccc}
\hline \hline$\sigma$ & Acc $(\%)$ & $S e(\%)$ & Training Time $(\mu \mathrm{s})$ & Testing Time $(\mu \mathrm{s})$ \\
\hline \hline 0.10 & $55.56 \pm 0.87$ & $33.33 \pm 0.92$ & $85 \pm 42$ & $42 \pm 3$ \\
0.20 & $57.78 \pm 1.01$ & $36.67 \pm 0.98$ & $86 \pm 39$ & $43 \pm 2$ \\
0.30 & $68.89 \pm 0.91$ & $56.00 \pm 1.05$ & $90 \pm 40$ & $42 \pm 3$ \\
0.40 & $70.67 \pm 1.18$ & $66.67 \pm 1.10$ & $91 \pm 41$ & $41 \pm 1$ \\
0.50 & $95.56 \pm 0.71$ & $93.33 \pm 0.81$ & $85 \pm 35$ & $43 \pm 2$ \\
0.60 & $95.56 \pm 0.55$ & $93.33 \pm 0.58$ & $86 \pm 37$ & $40 \pm 3$ \\
0.70 & $96.89 \pm 0.42$ & $95.33 \pm 0.64$ & $83 \pm 42$ & $38 \pm 2$ \\
0.80 & $97.78 \pm 0.53$ & $96.67 \pm 0.53$ & $81 \pm 38$ & $38 \pm 1$ \\
0.90 & $98.02 \pm 0.46$ & $97.78 \pm 0.41$ & $80 \pm 36$ & $37 \pm 2$ \\
\hline \hline
\end{tabular}

Table 2: Accuracy (Acc), sensitivity (Se) and positive predictive value (PPV) obtained by the OPF, SVM and MLP based classifiers from the features extracted by the GLCM method.

\begin{tabular}{lccc}
\hline \multicolumn{4}{c}{ GLCM } \\
\hline \hline Method & PPV $(\%)$ & $\mathrm{Se}(\%)$ & Acc(\%) \\
\hline \hline OPF-Euclidean & $97.00 \pm 0.05$ & $94.00 \pm 0.05$ & $96.00 \pm 0.03$ \\
OPF-Squared Chi-Sq. & $92.00 \pm 0.03$ & $84.00 \pm 0.04$ & $89.33 \pm 0.03$ \\
OPF-Manhattan & $90.00 \pm 0.05$ & $80.00 \pm 0.05$ & $86.67 \pm 0.03$ \\
OPF-Canberra & $90.00 \pm 0.04$ & $80.00 \pm 0.05$ & $86.67 \pm 0.00$ \\
OPF-Gaussian & $87.00 \pm 0.04$ & $74.00 \pm 0.05$ & $82.67 \pm 0.03$ \\
OPF-Chi-Square & $72.00 \pm 0.07$ & $44.00 \pm 0.04$ & $62.67 \pm 0.03$ \\
OPF-BrayCurtis & $62.00 \pm 0.07$ & $24.00 \pm 0.07$ & $49.33 \pm 0.04$ \\
SVM-Linear & $95.95 \pm 0.04$ & $91.89 \pm 0.04$ & $94.59 \pm 0.02$ \\
SVM-RBF & $95.54 \pm 0.87$ & $91.08 \pm 1.73$ & $94.05 \pm 1.15$ \\
SVM-Poly & $94.86 \pm 1.99$ & $89.73 \pm 3.97$ & $93.15 \pm 2.65$ \\
SVM-Sigmoid & $67.30 \pm 4.86$ & $34.59 \pm 9.73$ & $56.40 \pm 6.49$ \\
MLP 4/3/3 & $91.49 \pm 11.18$ & $82.97 \pm 22.35$ & $88.65 \pm 14.90$ \\
MLP 4/10/3 & $90.95 \pm 8.86$ & $81.89 \pm 17.72$ & $87.93 \pm 11.82$ \\
\hline \hline
\end{tabular}

Table 3: Accuracy (Acc), sensitivity (Se) and positive predictive value (PPV) obtained by the OPF, SVM and MLP based classifiers from the features extracted by SIM method.

\begin{tabular}{lccc}
\hline \multicolumn{4}{c}{ SIM } \\
\hline Method & PPV(\%) & Se(\%) & Acc(\%) \\
\hline \hline OPF-Gaussian & $98.00 \pm 0.04$ & $96.00 \pm 0.04$ & $97.53 \pm 0.05$ \\
OPF-Euclidean & $98.00 \pm 0.00$ & $96.00 \pm 0.00$ & $97.33 \pm 0.05$ \\
OPF-Squared Chi-Sq. & $97.00 \pm 0.05$ & $94.00 \pm 0.04$ & $96.00 \pm 0.05$ \\
OPF-Manhattan & $94.00 \pm 0.05$ & $88.00 \pm 0.05$ & $92.00 \pm 0.05$ \\
OPF-Canberra & $96.00 \pm 0.04$ & $92.00 \pm 0.05$ & $94.67 \pm 0.04$ \\
OPF-Chi-Square & $75.00 \pm 0.06$ & $50.00 \pm 0.06$ & $66.67 \pm 0.06$ \\
OPF-BrayCurtis & $71.00 \pm 0.06$ & $42.00 \pm 0.06$ & $61.33 \pm 0.06$ \\
SVM-Poly & $98.11 \pm 1.73$ & $96.22 \pm 3.46$ & $97.48 \pm 2.31$ \\
SVM-Linear & $97.30 \pm 0.55$ & $94.59 \pm 0.32$ & $96.40 \pm 0.32$ \\
SVM-RBF & $97.30 \pm 0.07$ & $94.59 \pm 0.07$ & $96.40 \pm 0.06$ \\
SVM-Sigmoid & $68.92 \pm 0.05$ & $37.84 \pm 0.05$ & $58.56 \pm 0.05$ \\
MLP 3/10/3 & $91.08 \pm 15.35$ & $82.16 \pm 30.70$ & $88.11 \pm 20.47$ \\
MLP 3/3/3 & $87.16 \pm 15.98$ & $74.32 \pm 31.96$ & $82.88 \pm 21.31$ \\
\hline \hline
\end{tabular}

test and total times are also indicated additionally to the average classification accuracy.

The Friedman test is useful to prove the null hypothesis $\left(H_{0}\right)$. Table 5 indicates the Friedman test results for the best classifier in combination with the features extractors in evaluation. The used confidence interval was equal to 0.001 , which represents the probability that
$H_{0}$ is incorrect, or the accuracy arrangement between features extractor methods/classifiers are not equivalent. Equivalence $(\square)$ among the results shows how efficient is the proposed approach when compared against other extractors already established in the literature. Superiority $(\triangle)$ or inferiority $(\nabla)$ mean that the proposed approach presents effectiveness higher or lower 
Table 4: Accuracy (Acc), sensitivity (Se) and positive predictive value (PPV) obtained by the OPF, SVM and MLP based classifiers from the features extracted by the SIM method plus the ones extracted by the VIF method.

\begin{tabular}{lccc}
\hline \multicolumn{4}{c}{ SIM+VIF } \\
\hline Method & PPV $(\%)$ & Se $(\%)$ & Acc $(\%)$ \\
\hline \hline OPF-Gaussian & $97.60 \pm 0.04$ & $95.20 \pm 0.08$ & $98.20 \pm 0.05$ \\
OPF-Euclidean & $97.01 \pm 0.01$ & $94.00 \pm 0.02$ & $96.00 \pm 0.01$ \\
OPF-Squared Chi-Sq. & $97.00 \pm 0.03$ & $94.00 \pm 0.03$ & $96.00 \pm 0.02$ \\
OPF-Manhattan & $97.00 \pm 0.02$ & $94.00 \pm 0.01$ & $96.00 \pm 0.01$ \\
OPF-Canberra & $97.00 \pm 0.02$ & $94.00 \pm 0.02$ & $96.00 \pm 0.01$ \\
OPF-Chi-Square & $80.00 \pm 0.07$ & $60.00 \pm 0.08$ & $73.33 \pm 0.07$ \\
OPF-BrayCurtis & $70.00 \pm 0.15$ & $40.00 \pm 0.18$ & $60.00 \pm 0.17$ \\
SVM-Poly & $97.70 \pm 0.87$ & $95.41 \pm 1.73$ & $96.94 \pm 1.15$ \\
SVM-Linear & $97.84 \pm 1.62$ & $95.68 \pm 3.24$ & $97.12 \pm 2.16$ \\
SVM-RBF & $98.65 \pm 0.08$ & $97.30 \pm 0.08$ & $98.20 \pm 0.07$ \\
SVM-Sigmoid & $68.92 \pm 0.11$ & $37.84 \pm 0.12$ & $58.56 \pm 0.09$ \\
MLP 4/10/3 & $91.35 \pm 12.58$ & $82.70 \pm 25.16$ & $88.47 \pm 16.77$ \\
MLP 4/3/3 & $87.57 \pm 12.90$ & $75.14 \pm 25.80$ & $83.42 \pm 17.20$ \\
\hline \hline
\end{tabular}

Table 5: Results of the Friedman's test on the Acc values achieved by the classifiers in combination with the results obtained in the classification of the experimental image dataset used by the classifiers under comparison ( $\square$ - Equivalence, $\triangle$ - Superiority, $\nabla$ - Inferiority.)

\begin{tabular}{|c|c|c|c|c|c|}
\hline Method & Acc (\%) & Statistical test & Training time $(\mu \mathrm{s})$ & Testing time $(\mu \mathrm{s})$ & Total time $(\mu \mathrm{s})$ \\
\hline \multicolumn{6}{|c|}{ GLCM } \\
\hline OPF-Euclidean & $96.00 \pm 0.03$ & $\triangle$ & $82 \pm 63$ & $124 \pm 19$ & $206 \pm 42$ \\
\hline SVM-Linear & $94.59 \pm 0.02$ & $\triangle$ & $7389 \pm 1320$ & $4024 \pm 697$ & $11413 \pm 1050$ \\
\hline MLP $4 / 3 / 3$ & $88.65 \pm 14.90$ & $\triangle$ & $53336 \pm 38700$ & $1 \pm 1$ & $53337 \pm 38799$ \\
\hline \multicolumn{6}{|c|}{$\overline{\mathrm{SIM}}$} \\
\hline OPF-Gaussian & $97.53 \pm 0.05$ & $\bar{\triangle} \triangle$ & $86 \pm 42$ & $\overline{37 \pm 1}$ & $123 \pm 43$ \\
\hline SVM-Poly & $97.48 \pm 2.31$ & $\triangle$ & $200294 \pm 32606$ & $444 \pm 481$ & $200738 \pm 33485$ \\
\hline MLP $4 / 10 / 3$ & $88.11 \pm 20.47$ & $\triangle$ & $89800 \pm 27442$ & $1 \pm 1$ & $89801 \pm 27441$ \\
\hline \multicolumn{6}{|c|}{ SIM+VIF } \\
\hline OPF-Gaussian & $\overline{98.20} \pm 0.05$ & - & $\overline{80 \pm 36}$ & $\overline{37 \pm 2}$ & $\mathbf{1 1 7} \pm \mathbf{3 9}$ \\
\hline SVM-RBF & $98.20 \pm 0.07$ & $\square$ & $15085 \pm 3268$ & $3552 \pm 5402$ & $18637 \pm 8670$ \\
\hline MLP 4/10/3 & $88.47 \pm 16.77$ & $\triangle$ & $77983 \pm 30314$ & $1 \pm 1$ & $77984 \pm 30315$ \\
\hline
\end{tabular}

than the method in comparison, respectively. From the data presented in Table 5, one can realize that the proposed approach based on SIM+VIF features combined with OPF and the Gaussian distance performs similarly to the SVM classifier with RBF kernel applied on SIM+VIF features, but is higher than the other approaches based on GLCM and SIM features. Also, the fastest classifier to train was the OPF classifier with Gaussian distance, the fastest to test was the MLP classifier, and the one that takes the lowest total time was the OPF classifier with Gaussian distance. Compared against the SVM-RBF classifier, the proposed approach had lower training and test times, thus presenting itself as the most efficient solution among the ones under comparison.

From the results obtained, it is possible to conclude that the best classification accuracy achieved from the features extracted by the GLCM method was obtained by the OPF classifier with Euclidean distance, which had also the lowest training and total times. On the other hand, the best classification accuracy obtained from the features extracted by the SIM method was achieved using the OPF classifier with the Gaussian distance. Additionally, this classifier had also in this case the lowest training and total execution times. The OPF classifier with the Gaussian distance achieved the best classification accuracy achieved from the SIM+VIF features, which was equal to $98.20 \%$; in addition, this configuration led to the lowest total time.

Another analysis that can be performed is based on the confusion matrix presented in Table 6 , which depicts the classes under study and the obtained classification results according to the classifier and the feature extraction method. Analyzing the data in Table 6, it can be easily noticed the low accuracies obtained by the MLP based classifier, which is indicated by many errors verified in the confusion matrix. It can also be noted that the OPF and SVM based classifiers successfully 
Table 6: Average confusion matrices for 10 runnings using the best classifiers under comparison applied on the features extracted using the GLCM and SIM methods and their combination (VIF + SIM).

\begin{tabular}{|c|c|c|c|c|}
\hline \multicolumn{5}{|c|}{ GLCM } \\
\hline True Class & Classified as & OPF-Euclidean & SVM-Linear & $\operatorname{MLP} 4 / 3 / 3$ \\
\hline \multirow{3}{*}{ Normal } & Normal & 13 & 12 & 12.7 \\
\hline & Fibrosis & 1 & 2 & 0.7 \\
\hline & COPD & 0 & 0 & 0.6 \\
\hline \multirow{3}{*}{ Fibrosis } & Normal & 0.5 & 2 & 1.8 \\
\hline & Fibrosis & 10.5 & 9 & 8.3 \\
\hline & COPD & 0 & 0 & 0.9 \\
\hline \multirow{3}{*}{ COPD } & Normal & 0 & 0 & 1.1 \\
\hline & Fibrosis & 0 & 0 & 1.2 \\
\hline & COPD & 12 & 12 & 9.7 \\
\hline \multicolumn{5}{|c|}{ SIM } \\
\hline True Class & Classified as & OPF-Gaussian & SVM-Polynomial & MLP 4/10/3 \\
\hline \multirow{3}{*}{ Normal } & Normal & 13.5 & 13.6 & 10.7 \\
\hline & Fibrosis & 0.5 & 0.4 & 2.1 \\
\hline & COPD & 0 & 0 & 1.2 \\
\hline \multirow{3}{*}{ Fibrosis } & Normal & 0 & 0.4 & 1.8 \\
\hline & Fibrosis & 11 & 10.6 & 8.8 \\
\hline & COPD & 0 & 0 & 0.4 \\
\hline \multirow{3}{*}{ COPD } & Normal & 0 & 0 & 1.1 \\
\hline & Fibrosis & 0 & 0 & 0.7 \\
\hline & COPD & 12 & 12 & 10.2 \\
\hline \multicolumn{5}{|c|}{$\mathrm{SIM}+\mathrm{VIF}$} \\
\hline True Class & Classified as & OPF-Gaussian & SVM-RBF & MLP $4 / 10 / 3$ \\
\hline \multirow{3}{*}{ Normal } & Normal & 13.8 & 13.5 & $\overline{12}$ \\
\hline & Fibrosis & 0.2 & 0.5 & 1.2 \\
\hline & COPD & 0 & 0 & 0.8 \\
\hline \multirow{3}{*}{ Fibrosis } & Normal & 0 & 0.1 & 1.6 \\
\hline & Fibrosis & 11 & 10.9 & 7.6 \\
\hline & COPD & 0 & 0 & 1.8 \\
\hline \multirow{3}{*}{ COPD } & Normal & 0 & 0 & 3 \\
\hline & Fibrosis & 0 & 0 & 2.2 \\
\hline & COPD & 12 & 12 & 9 \\
\hline
\end{tabular}

classified the COPD class, as there is no error in their classification results. The same did not occur for the Fibrosis and Normal classes, as some errors occurred with both classifiers.

Based on the experimental findings, it should be stressed that the OPF classifier with the Gaussian distance achieved no error in the classification of the Fibrosis class, both from the SIM features and from the SIM features plus the VIF features. It should also be noted that the classifier that obtained fewer classification errors as to the normal class was the OPF classifier with the Euclidean distance when applied on the SIM+VIF features.

\section{Conclusion}

In this article, an assessment of the OPF classifier performance to handle the task of lung disease diagnosis in CT images was presented. In this evaluation, three feature extraction methods and seven distance metric functions were employed and compared.

In particular, the OPF classifier configured with the Gaussian distance achieved very satisfactory accuracy levels, both when applied on the features extracted using the GLCM method and on the ones extracted using the SIM method. It can also be noted an accuracy gain of $2.2 \%$ relatively to the results presented in 16 using 
the same experimental image dataset. Among the extraction methods, SIM led to higher accuracies, mainly when associated with the VIF metric and the proposed Gaussian distance with the OPF classifier.

Based on the results obtained from the experiments conducted, one can conclude that as to the COPD class, the OPF and SVM classifiers with the features extracted using the SIM and VIF methods were the most promising ones, without classification errors. Relatively to the Fibrosis class, the OPF classifier with the Gaussian distance had no classification errors from the features extracted using the SIM method, neither from the features extracted using the SIM method plus the ones using the VIF method.

Relatively to the other traditional supervised learning algorithms studied here, namely, SVM and MLP, the OPF classifier confirmed its classification suitability, which was assessed in terms of accuracy, precision, recall and $F$-measure, as well as its superior computational efficiency, being therefore a very promising tool to identify pulmonary diseases from CT images.

\section{Acknowledgements}

The authors thank the Graduate Program in Computer Science from the Federal Institute of Education, Science and Technology of Ceará and the Department of Computer Engineering from the Walter Cantídio University Hospital of the Federal University of Ceará, in Brazil, for the support given.

The first author acknowledges the sponsorship from the Federal Institute of Education, Science and Technology of Ceará through grants PROINFRA/2013 and PROAPP/2014. The author acknowledges also the sponsorship from the Brazilian National Council for Research and Development (CNPq).

Victor Hugo C. de Albuquerque thanks CNPq for providing financial support through grants 470501/20138 and 301928/2014-2.

João P. Papa is grateful to São Paulo Research Foundation grants \#2014/16250-9 and \#2014/12236-1, as well as CNPq grant \#306166/2014-3.

Authors gratefully acknowledge the funding of Project NORTE-01-0145-FEDER-000022 - SciTech - Science and Technology for Competitive and Sustainable Industries, cofinanced by "Programa Operacional Regional do Norte" (NORTE2020), through "Fundo Europeu de Desenvolvimento Regional" (FEDER).

\section{Conflict of interest statement}

The authors report no conflict of interest.

\section{References}

1. WHO. Causes of death in the world. Technical report, World Health Organization, 2016.

2. Marcelo Alcantara Holanda, Ricardo Coelho Reis, Georgia Freire Paiva Winkeler, Simone Castelo Branco Fortaleza, José Wellington Oliveira Lima, and Eanes Delgado Barros Pereira. Influence of total face, facial and nasal masks on shortterm adverse effects during noninvasive ventilation. Jornal Brasileiro de Pneumologia, 35:164 - 173, 02 2009.

3. M. A. Holanda, A. C. Fortaleza, M. A. de Almeida, G. F. Winkeler, R. C. Reis, J. H. Hebert, J. W. Lima, and E. D. Pereira. Continuous positive airway pressure effects on regional lung aeration in patients with COPD: a high-resolution CT scan study. Chest, 138(2):305-14, 2010.

4. Peter J Barnes. Immunology of asthma and chronic obstructive pulmonary disease. Nature Reviews Immunology, 8(3):183-192, 2008.

5. WHO. Chronic obstructive pulmonary disease (copd). Technical report, World Health Organization, 2016.

6. R. de Marco, S. Accordini, I. Cerveri, A. Corsico, J. Sunyer, F. Neukirch, N. Kunzly, B. Leynaert, C. Janson, T. Gislason, P. Vermeire, C. Svanes, J.M. Anto, and P. Burney. An international survey of chronic obstrutive pulmonary disease in young adults according to gold stages. Thorax, 59:120 125, Julho 2004.

7. P. P. Rebouças Filho, P. C. Cortez, J. H. S. Felix, Tarique da Silveira T. S. Cavalcante, and M. A. Holanda. 3D segmentation and visualization of lung and its structures using CT images of the thorax. Journal of Biomedical Science and Engineering, pages 1099-1108, 2013.

8. P. P. Rebouças Filho, P. C. Cortez, J. H. S. Felix, Tarique da Silveira T. S. Cavalcante, and M. A. Holanda. Adaptive 2D crisp active contour model applied to lung segmentation in CT images of the thorax of healthy volunteers and patients with pulmonary emphysema. Revista Brasileira de Engenharia Biomédica, pages 363-376, 2013.

9. E. Souza Rebouças, R. Moura Sarmento, and P. Pedrosa Reboucas Filho. 3D adaptive balloon active contour: method of segmentation of structures in three dimensions. IEEE Latin America Transactions, 13(1):195-203, Jan 2015.

10. E. Cavalcanti Neto, P. Cesar Cortez, T. Silveira Cavalcante, V. Enoc Rodrigues, P. Pedrosa Reboucas Filho, and M. Alcantara Holanda. 3D lung fissure segmentation in TC images based in textures. 
IEEE Latin America Transactions, 14(1):254-258, Jan 2016.

11. Tan Kok Liang, Toshiyuki Tanaka, Hidetoshi Nakamura, Toru Shirahata, and Hiroaki Sugiura. An automated 3D emphysema extraction method using lung CT. SICE Annual Conference 2008, pages 3110-3114, August 2008.

12. Zhen Ma, J. M. R. S. Tavares, Renato Natal Jorge, and T. Mascarenhas. A review of algorithms for medical image segmentation and their applications to the female pelvic cavity. Computer Methods in Biomechanics and Biomedical Engineering, 13(2):235-246, 2010.

13. Pedro Pedrosa Rebouças Filho, Paulo César Cortez, Antônio C. da Silva Barros, Victor Hugo C. Albuquerque, and João Manuel R. S. Tavares. Novel and powerful 3D adaptive crisp active contour method applied in the segmentation of CT lung images. Medical Image Analysis, 35:503 - 516, 2017.

14. Zhen Ma, J. Manuel R. S. Tavares, and R. M. Natal Jorge. A review on the current segmentation algorithms for medical images. 1st International Conference on Imaging Theory and Applications (IMAGAPP), 5(8):135-140, 2009.

15. R.M. Haralick, K. Shanmugam, and Its'Hak Dinstein. Textural features for image classification. IEEE Transactions on Systems, Man and Cybernetics, SMC-3(6):610-621, Nov 1973.

16. G. L. B. Ramalho, P. P. Rebouças Filho, Fatima Nelsizeuma S. de Medeiros, and P. C. Cortez. Lung disease detection using feature extraction and extreme learning machine. Revista Brasileira de Engenharia Biomédica, 30:363-376, 2014.

17. M. Costa Oliveira and J. Raniery Ferreira. A bagof-tasks approach to speed up the lung nodules retrieval in the bigdata age. In IEEE 15th International Conference o e-Health Networking, Applications Services (Healthcom), pages 632-636, Oct 2013.

18. John Hebert Silva Felix, Paulo César Cortez, Pedro Pedrosa RebouçasFilho, Auzuir Ripardo de Alexandria, Rodrigo Carvalho Sousa Costa, and Marcelo Alcantara Holanda. Identification and quantification of pulmonary emphysema through pseudocolors. Lecture Notes in Computer Science, 5317:957-964, 2008.

19. H.R. Sheikh, A.C. Bovik, and G. de Veciana. An information fidelity criterion for image quality assessment using natural scene statistics. IEEE Transactions on Image Processing, 14(12):2117-2128, Dec 2005.
20. J. P. Papa, A. X. Falcão, and Celso T. N. Suzuki. Supervised pattern classification based on Optimum-Path Forest. International Journal of Imaging Systems and Technology, 19(2):120-131, 2009.

21. J. P. Papa, V. H. C. Albuquerque, A. X. Falcão, and J. M. R. S. Tavares. Efficient supervised OptimumPath Forest classification for large datasets. Pattern Recognition, 45:512-520, 2012.

22. J. P. Papa, S. E. N. Fernandes, and A. X. Falcão. Optimum-path forest based on k-connectivity: Theory and applications. Pattern Recognition Letters, $87: 117-126,2017$.

23. Thiago M. Nunes, Andre L.V. Coelho, Clodoaldo A.M. Lima, Joao P. Papa, and V. H. C. Albuquerque. EEG signal classification for epilepsy diagnosis via optimum path forest - a systematic assessment. Neurocomputing, 136:103-123, 2014.

24. Eduardo Jose da S. Luz, Thiago M. Nunes, V. H. C. Albuquerque, João P. Papa, and David Menotti. ECG arrhythmia classification based on OptimumPath Forest. Expert Systems with Applications, 40(9):3561-3573, 2013.

25. C. Suzuki, J. Gomes, A. Falcão, J. P. Papa, and S. Hoshino-Shimizu. Automatic segmentation and classification of human intestinal parasites from microscopy images. IEEE Transactions on Biomedical Engineering, 60(9):803-812, 2012.

26. FÁbio A.M. Capabianco, Alexandre X. Falcão, Clarissa L. Yasuda, and Jayaram K. Udupa. Brain tissue MR-image segmentation via optimum-path forest clustering. IEEE Transactions on Image Processing, 116:1047-1059, 2012.

27. A. I. Iliev, M. S. Scordilis, J. P. Papa, and A. X. Falcão. Spoken emotion recognition through Optimum-path Forest classification using glottal features. Computer Speech \& Language, 24:445460, 2010.

28. J. P. Papa, A. X. Falcão, G. M. Freitas, and A. M. H. Ávila. Robust pruning of training patterns for Optimum-Path Forest classification applied to satellite-based rainfall occurrence estimation. IEEE Geoscience and Remote Sensing Letters, 7(2):396400, 2010.

29. J. P. Papa, R. Y. M. Nakamura, V. H. C. Albuquerque, A. X. Falcão, and J. M. R. S. Tavares. Computer techniques towards the automatic characterization of graphite particles in metallographic images of industrial materials. Expert Systems with Applications, 40:590-597, 2013.

30. C. C. O. Ramos, A. N. Souza, J. P. Papa, and A. X. Falcão. A new approach for nontechnical losses detection based on Optimum-Path Forest. IEEE 
Transactions on Power Systems, 26:181-189, 2011.

31. C. R. Pereira, R. Y. M. Nakamura, K. A. P. Costa, and J. P. Papa. An Optimum-Path Forest framework for intrusion detection in computer networks. Engineering Applications of Artificial Intelligence, 25:1226-1234, 2012.

32. A. T. Silva, A. X. Falcão, and L. P. Magalhães. Active learning paradigms for CBIR systems based on Optimum-path Forest classification. Pattern Recognition, 44:2971-2978, 2011.

33. Thiago M. Nunes, V. H. C. Albuquerque, Jo ao P. Papa, Cleiton C. Silva, Paulo G. Normando, Elineudo P. Moura, and J. Manuel R. S. Tavares. Automatic microstructural characterization and classification using artificial intelligence techniques on ultrasound signals. Expert Systems with Applications, 40:3096-3105, 2013.

34. Samuel L. Gomes, Elizângela de S. Rebouças, Edson Cavalcanti Neto, João P. Papa, Victor H. C. de Albuquerque, Pedro P. Rebouças Filho, and João Manuel R. S. Tavares. Embedded real-time speed limit sign recognition using image processing and machine learning techniques. Neural Computing and Applications, pages 1-12, 2016.

35. Edgard M. Silva, Leandro B. Marinho, Pedro P. Rebouças Filho, João P. Leite, Josinaldo P. Leite, Walter M. L. Fialho, Victor Hugo C. de Albuquerque, and João Manuel R. S. Tavares. Classification of induced magnetic field signals for the microstructural characterization of sigma phase in duplex stainless steels. Metals, 6(7):164, 2016.

36. Edson Cavalcanti Neto, Samuel Luz Gomes, Pedro Pedrosa Rebouças Filho, and Victor Hugo C. de Albuquerque. Brazilian vehicle identification using a new embedded plate recognition system. Measurement, $70: 36$ - 46, 2015.

37. Victor Hugo C. de Albuquerque, Cleisson V. Barbosa, Cleiton C. Silva, Elineudo P. Moura, Pedro P. Rebouças Filho, João P. Papa, and João Manuel R. S. Tavares. Ultrasonic sensor signals and optimum path forest classifier for the microstructural characterization of thermally-aged inconel 625 alloy. Sensors, 15(6):12474, 2015.

38. A.S. Iwashita, J.P. Papa, A.N. Souza, A.X. Falcão, R.A. Lotufo, V.M. Oliveira, Victor Hugo C. de Albuquerque, and João Manuel R.S. Tavares. A pathand label-cost propagation approach to speedup the training of the optimum-path forest classifier. Pattern Recognition Letters, 40:121 - 127, 2014.

39. Thiago M. Nunes, André L.V. Coelho, Clodoaldo A.M. Lima, João P. Papa, and Victor Hugo C. de Albuquerque. EEG signal classification for epilepsy diagnosis via optimum path forest - a systematic assessment. Neurocomputing, 136:103 $123,2014$.

40. T. M. Nunes, V. H. C. Albuquerque, J. P. Papa, C. S. Silva, P. G. Normando, E. P. Moura, and J. M. R. S. Tavares. Automatic microstructural characterization and classification using artificial intelligence techniques on ultrasound signals. Expert Systems with Applications, 40(8):3096-3105, 2013.

41. Hjalmar K. Turesson, Sidarta Ribeiro, Danillo R. Pereira, João P. Papa, and Victor Hugo C. de Albuquerque. Machine learning algorithms for automatic classification of marmoset vocalizations. PLoS ONE, 11(9):1-14, 092016.

42. Victor Hugo C. de Albuquerque, Thiago M. Nunes, Danillo R. Pereira, Eduardo José da S. Luz, David Menotti, João P. Papa, and João Manuel R. S. Tavares. Robust automated cardiac arrhythmia detection in ECG beat signals. Neural Computing and Applications, pages 1-15, 2016.

43. Eduardo José da S. Luz, Thiago M. Nunes, Victor Hugo C. de Albuquerque, João P. Papa, and David Menotti. ECG arrhythmia classification based on optimum-path forest. Expert Systems with Applications, 40(9):3561 - 3573, 2013.

44. A. T. Silva, J. A. Santos, A.X. Falcão, R. S. Torres, and L. P. Magalhães. Incorporating multiple distance spaces in Optimum-Path Forest classification to improve feedback-based learning. Computer Vision and Image Understanding, 116:510-523, 2012.

45. Auzuir Ripardo De Alexandria, Paulo César Cortez, Jessyca Almeida Bessa, John Hebert da Silva Félix, José Sebastião De Abreu, and Victor Hugo C De Albuquerque. psnakes: A new radial active contour model and its application in the segmentation of the left ventricle from echocardiographic images. Computer methods and programs in biomedicine, 116(3):260-273, 2014.

46. Pedro Pedrosa Rebouças Filho, Paulo César Cortez, Antônio Carlos da Silva Barros, and Victor Hugo C De Albuquerque. Novel adaptive balloon active contour method based on internal force for image segmentation-a systematic evaluation on synthetic and real images. Expert Systems with Applications, 41(17):7707-7721, 2014.

47. Francisco Diego Lima Moreira, Maurício Nunes Kleinberg, Hemerson Furtado Arruda, Francisco Nélio Costa Freitas, Marcelo Monteiro Valente Parente, Victor Hugo Costa de Albuquerque, and Pedro Pedrosa Rebouças Filho. A novel vickers hardness measurement technique based on adaptive balloon active contour method. Expert Systems with Applications, 45:294-306, 2016. 
48. Pedro Pedrosa Rebouc, Roger Moura Sarmento, Paulo C Cortez, Victor Hugo C De, et al. Adaptive crisp active contour method for segmentation and reconstruction of $3 \mathrm{~d}$ lung structures. International Journal of Computer Applications, 111(4), 2015.

49. E. Cavalcanti Neto, P. C. Cortez, T. S. Cavalcante, V. E. R. da Silva Filho, P. P. Reboouç Filho, and M. A. Holanda. Supervised Enhancement Filter Applied to Fissure Detection, pages 337-340. Springer International Publishing, Cham, 2015.

50. Murillo B. Rodrigues, Leandro B. Marinho, Raul Victor M. Nóbrega, João Wellington M. Souza, and Pedro Pedrosa Rebouças Filho. Lung Segmentation in Chest Computerized Tomography Images Using the Border Following Algorithm, pages 539-548. Springer International Publishing, Cham, 2017.

51. Igor Rafael S Valente, Paulo César Cortez, Edson Cavalcanti Neto, José Marques Soares, Victor Hugo C de Albuquerque, and João Manuel RS Tavares. Automatic 3D pulmonary nodule detection in ct images: a survey. Computer methods and programs in biomedicine, 124:91-107, 2016.

52. J. P. Papa, C. T. N. Suzuki, and A. X. Falcão. LibOPF: A library for the design of Optimum-Path Forest classifiers, 2009. Software version 2.0 available at http://www.ic.unicamp.br/ afalcao/ LibOPF.

53. J. H. S. Felix, P. C. Cortez, M. A. Holanda, and R. C. S. Costa. Automatic segmentation and measurement of the lungs in healthy persons and in patients with chronic obstructive pulmonary disease in CT images. volume 18, pages 370-373, Margarita Island, Venezuela, October 2007. IV Latin American Congress on Biomedical Engineering 2007, Bioengineering Solutions for Latin America Health.

54. John Heber S. Felix, P. C. Cortez, M. A. Holanda, D. F. Colaço, V. H. C. Albuquerque, and A. R. Alexandria. Lung and chest wall structures segmentation in CT images. pages 291-294. Computational Vision and Medical Image Processing (VIPMAGE), Outubro 2007.

55. Pedro Pedrosa Rebouças Filho, Paulo César Cortez, and Marcelo Alcantara Holanda. Active contour modes crisp: new technique for segmentation the lungs in CT images. Revista Brasileira de Engenharia Biomédica, 27:259-272, 2011.

56. Geraldo L. Bezerra Ramalho, Daniel S. Ferreira, Pedro P. Rebouças Filho, and Fátima N. Sombra de Medeiros. Rotation-invariant feature extraction using a structural co-occurrence matrix. Measurement, 94:406 - 415, 2016.

57. Pedro P Rebouças Filho, Elizângela de S Rebouças, Leandro B Marinho, Róger M Sarmento, João
Manuel RS Tavares, and Victor Hugo C de Albuquerque. Analysis of human tissue densities: A new approach to extract features from medical images. Pattern Recognition Letters, 2017.

58. Ki-Young Choi and SS Kim. Morphological analysis and classification of types of surface corrosion damage by digital image processing. Corrosion Science, 47(1):1-15, 2005.

59. Fátima N S Medeiros, Geraldo L B Ramalho, Mariana P Bento, and Luiz CL Medeiros. On the evaluation of texture and color features for nondestructive corrosion detection. EURASIP Journal on Advances in Signal Processing, 2010:7, 2010.

60. A. Ultsch. $\mathrm{U}^{*}$-matrix: a tool to visualize clusters in high dimensional data. University of Marburg, Department of Computer Science, (36):1-10, 2003.

61. J. P. Papa, A. X. Falcão, and C. T. N. Suzuki. Supervised pattern classification based on optimumpath forest. International Journal of Imaging Systems and Technology, 19(2):120-131, 2009.

62. A.X. Falcão, J. Stolfi, and R.A. Lotufo. The Image Foresting Transform: Theory, Algorithms, and Applications. IEEE Transactions on Pattern Analysis and Machine Intelligence, 26(1):19-29, 2004.

63. C. Allène, J.Y. Audibert, M. Couprie, J. Cousty, and R. Keriven. Some links between min-cuts, optimal spanning forests and watersheds. In Proceedings of the 8th International Symposium on Mathematical Morphology, pages 253-264, 2007.

64. H. Liu, S. Jiang, Q. Huang, and C. Xu W. Gao region-based visual attention analysis with its application in image browsing on small displays. In Proceedings of the 15th international Conference on Multimedia, pages 25-29, September 2007.

65. Dennis W Ruck, Steven K Rogers, Matthew Kabrisky, Mark E Oxley, and Bruce W Suter. The multilayer perceptron as an approximation to a bayes optimal discriminant function. IEEE Transactions on Neural Networks, 1(4):296-298, 1990.

66. S. Nissen. Implementation of a Fast Artificial Neural Network Library (FANN), 2003. Department of Computer Science University of Copenhagen (DIKU). Software available at http://leenissen. $\mathrm{dk} / \mathrm{fann} /$.

67. Simon O Haykin. Neural Networks and Learning Machines. Pearson Prentice Hall, 2008.

68. C. Cortes and V. Vapnik. Support vector networks. Machine Learning, 20:273-297, 1995.

69. C. J. C. Burges. A tutorial on support vector machines for pattern recognition. Data Mining and Knowledge Discovery, 2:121-167, 1998.

70. Chih-Chung Chang and Chih-Jen Lin. Libsvm: A library for support vector machines. ACM Trans. 
Intell. Syst. Technol., 2(3):27:1-27:27, May 2011.

71. Sidney Siegel. Estatística não-paramétrica para as ciências do comportamento. Série Métodos de Pesquisa. Artmed, Porto Alegre, RS, 2 edition, 7 2006.

72. Mario F Triola et al. Introdução à estatística, volume 10. LTC, Rio de Janeiro, RJ, 2005. 